Dr. -Ing. Herbert Börner

Technische Hochschule Ilmenau:

Elektrotechnik/Nachrichtentechnik

Dr. sc. phil. Gisela Buchheim

Dr. rer. nat., Professor, Technische Universität Dresden:

Kapiteleinführungen

Dr.sc. oec. Thomas Hänseroth

Dozent, Technische Universität Dresden:

Bauingenieurwissenschaften

Dr. sc.phil. Alfred Kirpal

Dr. -Ing., Dozent, Technische Hochschule Ilmenau:

Elektrotechnik/Elektronik

Dr. sc. phil. Klaus Krug

Dr. rer. nat., Professor, Technische Hochschule

„Carl Schorlemmer« Leuna-Merseburg:

Verfahrenstechnik

Dr. sc. phil. Peter Lange

Dr. rer. nat., Friedrich-Schiller-Universität Jena:

Verfahrenstechnik/Silikattechnik

Dr.-Ing. Klaus Mauersberger

Technische Universität Dresden:

Maschineningenieurwissenschaften

Almut Mehlhorn

Technische Universität Dresden:

Bildbesorgung, Literaturverzeichnis, Register

Dr. sc. phil. Friedrich Naumann

Dr. oec., Technische Universität Karl-Marx-Stadt:

Informatik

Dr. phil. Siegfried H. Richter

Dipl.-Ing., Technische Universität Dresden:

Maschineningenieurwissenschaften/Fertigungstechnik

Dr. sc. phil. Peter Schubert

Dr.-Ing., Dozent, Technische Universität Dresden:

Elektrotechnik/Energietechnik

Dr. phil. Rainer Sennewald

Dipl.-Ing., Bergakademie Freiberg:

Montanwissenschaften

Dr. phil. habil. Rolf Sonnemann

Dr. rer. oec., Professor, Technische Universität Dresden:

Vorwort, Einleitung

Dr. phil. Bernhard Sorms

Dipl.-Chem., Technische Universität Dresden:

Verfahrenstechnik/Technische Chemie

Dr. rer. nat. habil. Otfried Wagenbreth

Dozent, Technische Universität Dresden:

Montanwissenschaften 


\section{Geschichte der}

\section{Technikwissenschaften}

herausgegeben von Gisela Buchheim und Rolf Sonnemann 
CIP-Titelaufnahme der Deutschen Bibliothek

Geschichte der Technikwissenschaften/hrsg. von Gisela Buchheim u. Rolf Sonnemann.

ISBN 978-3-0348-6153-3 ISBN 978-3-0348-6152-6 (eBook)

DOI 10.1007/978-3-0348-6152-6

NE: Buchheim, Gisela [Hrsg.]

Das Werk ist urheberrechtlich geschützt. Die dadurch begründeten Rechte, insbesondere des Nachdruckes, der Entnahme von Abbildungen, der Funksendung, der Wiedergabe auf photomechanischem oder ähnlichem Wege und der Speicherung in Datenverarbeitungsanlagen bleiben, auch bei nur auszugsweiser Verwertung, vorbehalten. Die Vergütungsansprüche des $\S 54$, Abs. 2 UrhG werden durch die »Verwertungsgesellschaft Wort« München, wahrgenommen.

(C) 1990 Springer Basel AG

Ursprünglich erschienen bei Edition Leipzig 1990

Softcover reprint of the hardcover 1st edition 1990

ISBN 978-3-0348-6153-3 


\section{Inhalt}

7 Vorwort

10 Einleitung

19 Technisches Wissen in der handwerklichen Produktion

19 Einführung

24 Anfänge des Bergbaus und Hüttenwesens und ihre literarische Überlieferung

27 Bautechnisches Wissen in Altertum und Mittelalter

42 Quellen und Wurzeln des mechanisch-technischen Wissens in Antike und Mittelalter

55 Kenntnisse über Stoffwandlungen

64 Technisches Wissen - Ansätze seiner rationalen Durchdringung

64 Einführung

69 Anfänge der Montanwissenschaften bis zur Gründung der ersten Bergakademien

91 Aufbruch zu den Bauingenieurwissenschaften

111 Praktische Mechanik und Maschinenkunde im Spannungsfeld von Naturwissenschaften und Technik

134 Chemische Gewerbe und richtungweisende Technologiekonzepte

145 Herausbildung der Technikwissenschaften in der industriellen Revolution

145 Einführung

151 Fachliche Komplettierung und regionale Verbreitung der Montanwissenschaften

163 Herausbildung der Bauingenieurwissenschaften in der Polarität von traditioneller und neuer Bautechnik

180 Von den mechanischen Künsten zum wissenschaftlichen Maschinenbau

203 Chemische Technologie

213 Voraussetzungen der wissenschaftlichen Elektrotechnik

221 Rechnen mit Maschinen 


\section{Technikwissenschaften und "große Industrie»}

228 Einführung

235 Neue Bergbauzweige und Spezialdisziplinen der Montanwissenschaften

245 Stahl- und Stahlbetonbau als Domänen der Bauingenieurwissenschaften

264 Konsolidierung der Maschinenwissenschaften in der Zeit aufkommender Massenfertigung

281 Empirische Verfahrensprinzipien in der chemischen Technologie

299 Starkstromtechnik und Schwachstromtechnik

323 Mechanische Datenverarbeitung

331 Technikwissenschaften und Rationalisierung in den zwanziger Jahren

331 Einführung

335 Weitere Spezialisierung und erste Integrationstendenzen in den Montanwissenschaften

340 Bauingenieurwissenschaften - Rationalisierung und Industrialisierung des Bauens

351 Wissenschaftliches Maschinenwesen unter den Bedingungen der kapitalistischen Rationalisierung

368 Herausbildung des Verfahrensingenieurwesens

382 Elektrotechnik in der Differenzierung

409 Wissenschaftliche Voraussetzungen für die Informatik

417 Technikwissenschaften in der wissenschaftlich-technischen Revolution

417 Einführung

420 Tendenzen des Maschinenwesens

437 Prozeß- und Systemverfahrenstechnik

449 Mikroelektronik auf dem Vormarsch

468 Informatik - neue Möglichkeiten der Datenverarbeitung

\section{Literatur (Auswahl)}

490 Bildnachweis

492 Register 


\section{Vorwort}

Die Geschichte der Technikwissenschaften gehörte noch vor zehn Jahren zu den Desiderata wissenschaftshistorischer Forschung. Von einer so bezeichneten Disziplin war kaum die Rede. Das nimmt nicht wunder, wenn man bedenkt, daß Maschinenbauer und Elektrotechniker, Bauingenieure und Verfahrenstechniker das von ihnen vertretene Gebiet entweder unter die angewandten Naturwissenschaften subsumierten oder es ganz allgemein der Technik zuordneten.

Mit diesem Buch legen wir das Ergebnis einer Gemeinschaftsarbeit vor, die vor etwa einem Jahrzehnt begann. Die Gründung eines Zentrums für Geschichte der Technikwissenschaften an der Technischen Universität Dresden geschah in der Absicht, dem oben bezeichneten Mangel abzuhelfen. Promovierte oder doch zumindest diplomierte Ingenieure und Naturwissenschaftler mehrerer technischer Hochschulen der DDR vereinten sich in dem Bemühen, ihr ausgeprägtes historisches Interesse in die Bahnen einer systematisch betriebenen wissenschafts- und technikhistorischen Forschung $\mathrm{zu}$ lenken. Lernend $\mathrm{zu}$ lehren und lehrend $\mathrm{zu}$ lernen stellte nicht geringe Anforderungen an jeden der künftigen Autoren, zu denen sowohl Hochschullehrer gehören, die mit der Gründung des Zentrums als Lehrkräfte in Erscheinung traten, als auch jene Nachwuchswissenschaftler, von denen einige inzwischen in den Kreis der Dozenten und Professoren eingetreten sind.

Da die Zahl der technikwissenschaftlichen Spezialdisziplinen unter den Bedingungen der mit der wissenschaftlich-technischen Revolution einhergehenden wissenschaftlichen Arbeitsteilung von Jahr zu Jahr zunimmt, andererseits nicht erwartet werden kann, daß die noch in den Anfängen stehende historische Forschung sofort auch das Gesamtgebiet der Technikwissenschaften in Augenschein nimmt, versteht es sich, daß unserer Darstellung der Charakter des Fragmentarischen anhaftet. Der Leser wird manche Merkmale des Vorläufigen entdecken. Man mag das bedauern, doch sollte berücksichtigt werden, daß jedem Produkt forschenden Bemühens ein mehr oder minder hohes Maß des noch nicht Abgeschlossenen eigen ist. Wichtig erschien uns, mit der historischen Skizzierung solcher Gebiete zu beginnen, die in der Geschichte als erste zum Substrat wissenschaftlichen Denkens geworden waren. Dazu gehören zweifellos das Montanwesen, bestimmte Bereiche der Verfahrenstechnik, das Maschinen- und das Bauwesen. Ihnen vor allem galt unsere Aufmerksamkeit, die sich auch deshalb den genannten Disziplinen zuwandte, weil deren Repräsentanten 
dem Dresdener Zentrum angehören. Schließlich glauben wir mit Elektrotechnik und Informatik zwei Disziplingruppen in ihrem historischen Werden beschrieben zu haben, die im Ensemble der Hoch- oder Schlüsseltechnologien gegenwärtig und sicher auch künftighin eine überaus bedeutsame Rolle spielen. Wenn andererseits weder die Luft- und Raumfahrt noch die Raketentechnik, ganz zu schweigen die Medizintechnik, die ja in vieler Hinsicht Erkenntnisse der modernen Technikwissenschaften in sich aufnimmt, der wissenschaftshistorischen Befragung unterzogen wurden, dann mag das anderen Wissenschafts- und Technikhistorikern Ansporn sein, sich diesen Gebieten zuzuwenden.

Nur zu erkunden, wann wissenschaftliches Denken sich der Technik zuwandte und wessen Erkenntnisse den Arbeitsmitteln neue Funktion und Gestalt vermittelten, darin konnte sich das Bestreben der Autoren nicht erschöpfen. Wissenschaft und Technik standen immer in einem Bezug zu den großen Fragen der jeweiligen Epoche. Über das Wohl und Wehe der Technik ist seit Jahrhunderten nachgedacht worden, und spätestens seit Erscheinen der ersten Dampflokomotiven stritt man um das Für und Wider der Herrschaft des Menschen über die Natur. Dem Ingenieur ist es oft nicht leicht gemacht worden, sich im Schichtenbau der Gesellschaft zu behaupten. Es bedurfte langer Auseinandersetzungen mit der staatlichen Bürokratie, um auch ihm akademische Ehren zuzuerkennen, und die Universitäten taten das ihre, um den Ingenieuren den Eintritt in ihre bevorrechteten Kreise zu verwehren. Gewiß sind diese Diskussionen heute ausgestanden. Eine auf die Technikwissenschaften bezogene historische Darstellung muß sich jedoch der Aufgabe unterziehen, deutlich werden zu lassen, wie der Beruf des Ingenieurs entstand, welche Antworten er auf welche Fragen gab und wie er damit Verantwortung gegenüber der Gesellschaft übernahm.

$\mathrm{Da}$ vorausgesetzt werden durfte, daß dem Adressaten dieses Buches die allgemeinen historischen Umstände, unter denen moderne Technik ins Leben trat und zu wirken begann, in ungleich höherem Maß bekannt sind als die Daten und Entwicklungstendenzen weit zurückliegender Epochen, wird man finden, daß, je näher die jeweilige Darstellung zur Zeitgeschichte steht, die entsprechenden Passagen um so knapper gehalten wurden. Es ist $\mathrm{zu}$ hoffen, daß den Autoren daraus kein Vorwurf gemacht wird.

Die nicht geringe Zahl der beteiligten Autoren brachte es mit sich, daß die Diktion der vollkommenen Einheitlichkeit entbehrt. Hier den glättenden Hobel anzusetzen sahen sich die Herausgeber nicht veranlaßt. Wohl aber fühlten sie sich verpflichtet, einer Disposition zu folgen, der allgemeine Verbindlichkeit zukam.

Herausgeber und Autoren halten sich nicht für so kompetent, daß sie annehmen durften, ohne den Rat und das klärende Gespräch mit Fachkundigen verschiedener Grundlagen- und Spezialdisziplinen auszukommen. Nicht allen Kolleginnen und Kollegen kann hier der Dank abgestattet werden. Die ungenannt Bleibenden mögen sich deshalb in den Kreis derer einbezogen fühlen, denen wir vor allem herzlich danken: den Professoren E.-J.Gießmann (Berlin) und W.Purkert (Leipzig) für ihre sehr gründlichen Gutachten, den Professoren H. Göldner, F. Holzweißig und H. Prochnow (Dresden), W. Arnold und W. Wächtler (Freiberg) für manche Diskussion, 
ohne deren Ergebnisse die Exaktheit der wissenschaftlichen Aussage in diesem und jenem Abschnitt nicht erreicht worden wäre, den Kollegen Dr. M. Ketting (Lengenfeld), Dr. W. Piersig (Annaberg-Buchholz), Dr. M. Meyer (Rostock) und Dr. D. Schneider (Magdeburg) für hilfreiche Unterstützung ebenso wie den Mitarbeitern des Bereichs Geschichte der Produktivkräfte an der Sektion Philosophie und Kulturwissenschaften der Technischen Universität Dresden. Unser Dank gilt gleichermaßen Direktor Dr. R. Slotta (Bochum) wie den Fachkollegen, die anläßlich des Symposiums des Internationalen Komitees für Geschichte der Technik in Dresden 1986 mit mutmachenden Beiträgen aufwarteten. Schließlich danken wir dem Verlag EDITION LEIPZIG für die freundliche Unterstützung unseres Vorhabens. 